\title{
The Association between Adenosine Triphosphate Binding Cassette B1 Cytosine 3435 Thymine Polymorphism and Pharmacoresistant Idiopathic Epilepsy: An Egyptian Study
}

\author{
Yosria A. Altaweel ${ }^{1}$, Amr E. Kamel ${ }^{1}$, Karam S. Amin ${ }^{1}$, Somia H. Abd-ALLAH ${ }^{2}$, Shaimaa A. Elaidy ${ }^{1, *}$ \\ ${ }^{1}$ Neurology Department, Zagazig University, Egypt \\ ${ }^{2}$ Medical biochemistry Department, Zagazig University, Egypt \\ *Corresponding author: shaimaaelaidy@yahoo.com
}

Received March 01, 2019; Revised May 02, 2019; Accepted May 20, 2019

\begin{abstract}
Background: P-glycoprotein (P-gp) is a drug efflux transporter present in the blood brain barrier whose function was suggested to be modified by a genetic polymorphism affecting ABCB1 C3435T gene at exon 26 . Overexpression was related to homozygous $C$ allele in some studies, however other studies in different ethnic populations showed relation to homozygous $\mathrm{T}$ allele, furthermore others failed to confirm any relation to resistance to therapeutic effects of AEDs. Aim of the study: Our aim is to find out the association between C3435T polymorphism and pharmacoresistance in idiopathic epilepsy in order to identify early the pharmacoresistant patients so we can select the proper AED and other proper therapeutic modalities. Patients and methods: Our case-control study was conducted on 44 idiopathic epileptic patients (22 drug-resistant and 22 drug-responsive epilepsy) and 44 healthy controls of comparable age and sex. Blood samples were obtained. Allele and genotype frequencies were evaluated between our study groups and their association with pharmacoresistance, some historical and semiology of epilepsy. Results: Our work revealed a lower risk of drug resistance in patients with the genotype CT (OR: 0.8) in comparison to genotypes CC and TT (OR: 1.2, 1 respectively) but the results were not statistically significant. However, significant association regarding daytime seizures was found with genotype CC in comparison to genotypes CT and TT. Conclusion: Daytime seizures were found to be more prevalent among those with CC genotype and this association was significant. The risk of drug resistant epilepsy was lower in patients with CT than those with CC and TT genotypes, but this association was not statistically significant in our study.
\end{abstract}

Keywords: idiopathic epilepsy, drug resistant epilepsy, polymorphism, ABCB1

Cite This Article: Yosria A. Altaweel, Amr E. Kamel, Karam S. Amin, Somia H. Abd-ALLAH, and Shaimaa A. Elaidy, "The Association between Adenosine Triphosphate Binding Cassette B1 Cytosine 3435 Thymine Polymorphism and Pharmacoresistant Idiopathic Epilepsy: An Egyptian Study.” International Journal of Clinical and Experimental Neurology, vol. 7, no. 1 (2019): 7-11. doi: 10.12691/ijcen-7-1-2.

\section{Introduction}

Epilepsy is a disease of the brain characterized by recurrent unprovoked seizures. It has serious impact on cognitive, behavior and social functions of the affected populations and represents a heavy burden on the community [1].

Idiopathic epilepsies are a major portion of epilepsies of suspected genetic component with no gross abnormalities on neuroimaging [2]. Up till now despite the appearance of wide spectrum and different mechanisms of action of antiepileptic drugs, about 33\% of epileptic individuals continue to experience seizures [3].

Several studies had tried to explain the cause of pharmacoresistance. Some focused on clinical factors such as underlying structural abnormalities, seizure semiology, interictal EEG findings to explain this phenomenon [4].
However other studies suggested that impaired transfer of antiepileptic drugs (AEDs) across blood brain barrier (BBB) might play a role [5].

P-glycoprotein (P-gp) is a drug efflux transporter encoded by ABCB1 (ATP binding cassette subfamily b member 1) [6], whose genetic polymorphism at the coding region C3435T on exon 26 might play a role regulating its expression at BBB. The overexpression prevents access of AEDs to epileptogenic zone in the brain [7]. However, results are inconsistent and needs further assessment targeting patients with comparable clinical status (same age group, same type of epilepsy syndrome).

Aim of the work: our aim was to investigate the association between ABCB1 C3435T gene polymorphism and the pharmacoresistance among idiopathic epileptic Egyptian population in order to identify early the pharmacoresistant patients so we can select the proper AED and other proper therapeutic modalities. 


\section{Patients and Methods}

Our case control study was conducted on 44 idiopathic epileptic patients subclassified into 2 equal subgroups (22 drug-responsive epilepsy and 22 drug-resistant epilepsy). The case group was age and sex matched with a control group comprised of 44 healthy persons. The study was approved by Institution Review Board at Zagazig university. Patients were recruited from internal section and outpatient clinic of Zagazig university hospitals. All patients had an established diagnosis of idiopathic epilepsy with age less than 30 years. Drug resistance was defined as failure to obtain complete seizure remission for at least one year with the usage of adjusted tolerable dose of the appropriate AED to the patient situation [8]. Drug responsiveness represented patients with no seizure recurrence for the same duration of one year [8]. Compliance was determined using Morisky medication adherence scale [9]. Patients with all causes of symptomatic epilepsies, significant psychiatric problems, history of drug abuse were excluded from our study.

All patients signed an informed consent to participate in the study. In all patients, the allele, genotype frequencies were determined as well as the age of onset, duration of therapy with AEDs, number of currently used AEDs, seizure semiology, interictal EEG findings. ABCB1 C3435T gene polymorphism was compared between our study groups and its association with some historical data, semiology of epilepsy as well as interictal EEG findings.

\subsection{Genotyping Procedure}

Genotyping of C3435T variants of the ABCB1 gene was performed by polymerase chain reaction-restriction fragment length polymorphism (PCR-RFLP) [10].

\section{Results}

\subsection{Demographic Data}

Demographic characteristics of the patients are presented in Table 1. Patients with drug responsive epilepsy had lower mean age than patients with drug resistant epilepsy (17.8 years VS 19.5 years respectively). Males are overrepresented among drug responsive group (54.5\%) but data were statistically nonsignificant. History of status epilepticus was significant among drug resistant epileptic patients at $\mathrm{P}$ value 0.04 . Also, those patients had significant lower age of onset, longer duration of therapy, higher number of seizures before receiving medications. There was a larger proportion of patients on polytherapy in the drug resistant group (91.0\%) compared with the drug responsive group $(9 \%)(\mathrm{P}<0.001)$. The AEDs administered to patients were phenytoin, phenobarbital, carbamazepine, valproate, oxcarbazepine, levetiracetam, lamotrigine, clonazepam and topiramate. Focal epilepsy was significantly prevalent among drug resistant group (P value 0.04). Normal interictal EEG and primary generalized epileptogenic EEG abnormalities were statistically more in the drug responsive group ( $\mathrm{P}$ value 0.04 and 0.02 respectively).

Table 1. Demographic and clinical characteristics of our groups of study:

\begin{tabular}{|c|c|c|c|c|c|}
\hline & & $\begin{array}{c}\text { Drug responsive } \\
\text { epilepsy }(n=22)\end{array}$ & $\begin{array}{l}\text { Drug resistant } \\
\text { epilepsy }(n=22)\end{array}$ & Control $(n=44)$ & $P$ value \\
\hline Patient age in years $($ mean $\pm S D)$ & & $17.8 \pm 8.2$ & $19.5 \pm 8.3$ & $18.2 \pm 8.1$ & 0.8 \\
\hline \multirow{2}{*}{ Gender } & Males & $12(54.5 \%)$ & $7(31.8 \%)$ & $18(40.9 \%)$ & \multirow{2}{*}{0.3} \\
\hline & Females & $10(45.5 \%)$ & $15(68.2 \%)$ & $26(59.1 \%)$ & \\
\hline \multirow{2}{*}{ Family history of epilepsy } & Positive & $3(13.6 \%)$ & $8(36.4 \%)$ & & \multirow{2}{*}{0.08} \\
\hline & Negative & $19(86.4 \%)$ & $14(63.6 \%)$ & & \\
\hline \multirow{2}{*}{ Consanguinity } & Positive & $8(36.4 \%)$ & $10(45.5 \%)$ & & \multirow{2}{*}{0.5} \\
\hline & Negative & $14(63.6 \%)$ & $12(54.5 \%)$ & & \\
\hline \multirow{2}{*}{ History of febrile seizures } & Positive & $1(4.5 \%)$ & $2(9.1 \%)$ & & \multirow{2}{*}{0.9} \\
\hline & Negative & $21(95.5 \%)$ & $20(90.9 \%)$ & & \\
\hline \multirow{2}{*}{ History of status epilepticus } & \begin{tabular}{|l|} 
Positive \\
\end{tabular} & $0(0 \%)$ & $5(22.7 \%)$ & & \multirow{2}{*}{$0.04 *$} \\
\hline & Negative & $22(100 \%)$ & $17(77.3 \%)$ & & \\
\hline Age of onset of $1^{\text {st }}$ seizures (mean $\left.\pm S D\right)$ & & $13.09 \pm 6.72$ & $8.8 .9 \pm 5.14$ & & $0.02 *$ \\
\hline \multirow{2}{*}{ Number of seizures before TTT } & $\leq 4$ & $19(86.4 \%)$ & $10(45.5 \%)$ & & \multirow{2}{*}{$0.04^{*}$} \\
\hline & $>4$ & $3(13.6 \%)$ & $12(54.5 \%)$ & & \\
\hline Duration of therapy (years) & & $3.04 \pm 3.01$ & $10.54 \pm 5.57$ & & $0.00^{*}$ \\
\hline \multirow{2}{*}{ Number of AEDs } & Mono & $20(91 \%)$ & $2(9 \%)$ & & \multirow{2}{*}{$0.001 *$} \\
\hline & Poly & $2(9 \%)$ & $20(91 \%)$ & & \\
\hline \multirow{2}{*}{ Epilepsy type } & Idiopathic generalized epilepsy & $22(100 \%)$ & $17(77.3 \%)$ & & \multirow{2}{*}{$0.04 *$} \\
\hline & Idiopathic focal epilepsy & $0(0.0 \%)$ & $5(22.7 \%)$ & & \\
\hline
\end{tabular}

${ }^{*} \mathrm{p}<0.05$ is statistically significant.

Table 2. ABCB1 C435T polymorphism in our study participants:

\begin{tabular}{|c|c|c|c|c|c|c|c|c|}
\hline \multirow[t]{2}{*}{ Variables } & \multicolumn{2}{|c|}{$\begin{array}{l}\text { Drug responsive epilepsy } \\
(n=22)\end{array}$} & \multicolumn{2}{|c|}{$\begin{array}{l}\text { Drug resistant epilepsy } \\
(n=22)\end{array}$} & \multicolumn{2}{|c|}{ control } & \multirow[t]{2}{*}{$\chi^{2}$} & \multirow[t]{2}{*}{$\mathbf{P}$} \\
\hline & NO & $\%$ & NO & $\%$ & NO & $\%$ & & \\
\hline $\begin{array}{l}\text { Genotypes: } \\
C C \\
C T \\
T T\end{array}$ & $\begin{array}{c}8 \\
10 \\
4\end{array}$ & $\begin{array}{l}36.3 \\
45.5 \\
18.2\end{array}$ & $\begin{array}{c}7 \\
11 \\
4\end{array}$ & $\begin{array}{l}31.8 \\
50.0 \\
18.2\end{array}$ & $\begin{array}{l}17 \\
14 \\
13\end{array}$ & $\begin{array}{l}38.7 \\
31.8 \\
29.5\end{array}$ & $\begin{array}{c}0.01 \\
0.09 \\
0.9\end{array}$ & $\begin{array}{l}0.9 \\
0.7 \\
0.3\end{array}$ \\
\hline $\mathrm{C}$ allele & 18 & 81.8 & 18 & 81.8 & 31 & 70.5 & 0.01 & 0.9 \\
\hline$T$ allele & 14 & 63.6 & 15 & 68.2 & 27 & 61.4 & 0.01 & 0.9 \\
\hline
\end{tabular}


Table 3. Odds ratio of ABCB1 C3435T polymorphism between drug responsive and drug resistant epilepsy

\begin{tabular}{|l|c|c|c|}
\hline Variables & Drug responsive epilepsy (n=22) & Drug resistant epilepsy (n=22) & Odds ratio (95\% CI) \\
\hline Genotypes: & & 7 & $1.2(0.35-4.3)$ \\
CC & 8 & 11 & $0.8(0.25-2.7)$ \\
$C T$ & 10 & 4 & 18 \\
$T T$ & 4 & 18 & $1(0.22-4.6)$ \\
\hline C allele & 18 & 15 & $0.22-4.6)$ \\
\hline T allele & 14 & $0.8(0.23-2.8)$ & \\
\hline
\end{tabular}

Table 4. Association between history data, temporal pattern, epilepsy syndrome and interictal EEG findings and ABCB1 polymorphism in our study participants:

\begin{tabular}{|c|c|c|c|c|c|c|c|c|}
\hline \multirow{3}{*}{ Variables } & \multicolumn{6}{|c|}{ ABCB1 polymorphism genotypes } & \multirow{3}{*}{$\chi^{2}$} & \multirow{3}{*}{$\mathbf{P}$} \\
\hline & \multicolumn{2}{|c|}{$\mathrm{CC}(\mathrm{n}=15)$} & \multicolumn{2}{|c|}{$\mathrm{CT}(\mathrm{n}=21)$} & \multicolumn{2}{|c|}{$\mathrm{TT}(\mathrm{n}=8)$} & & \\
\hline & No & $\%$ & No & $\%$ & No & $\%$ & & \\
\hline Family history: & & & & & & & & \\
\hline Positive & 4 & 26.7 & 4 & 19.0 & 3 & 37.5 & 1.1 & 0.5 \\
\hline Negative & 11 & 73.3 & 17 & 81.0 & 5 & 62.5 & & \\
\hline Family consanguinity: & & & & & & & & \\
\hline Positive & 7 & 46.7 & 8 & 38.1 & 3 & 37.5 & 0.3 & 0.8 \\
\hline Negative & 8 & 53.3 & 13 & 61.9 & 5 & 62.5 & & \\
\hline History of febrile seizures: & & & & & & & & \\
\hline Positive & 0 & 0.0 & 2 & 9.5 & 1 & 12.5 & 1.7 & 0.4 \\
\hline Negative & 15 & 100 & 19 & 90.5 & 7 & 87.5 & & \\
\hline History of status epilepticus: & & & & & & & & \\
\hline Positive & 15 & 100 & 17 & 81 & 7 & 85.5 & 1.4 & 0.2 \\
\hline Negative & 0 & 0 & 4 & 19 & 1 & 12.5 & & \\
\hline Age of onset of $1^{S T}$ seizure: & & & & & & & & \\
\hline$<12$ years & 10 & 35.7 & 13 & 46.5 & 5 & 17.9 & 0.1 & 0.9 \\
\hline$>12$ years & 5 & 31.3 & 8 & 50 & 3 & 18.7 & & \\
\hline No of seizures prior to $A E D s$ & & & & & & & & \\
\hline$\leq 4$ seizures & 9 & 60.0 & 12 & 57.1 & 8 & 100 & 1.9 & 0.2 \\
\hline$>4$ seizures & 6 & 40.0 & 9 & 42.9 & 0 & 0.0 & & \\
\hline Temporal pattern: & & & & & & & & \\
\hline Diurnal & 10 & 66.7 & 13 & 61.9 & 2 & 25.0 & 3.7 & $0.05^{*}$ \\
\hline Nocturnal & 1 & 6.7 & 2 & 9.5 & 2 & 25.0 & 0.7 & 0.3 \\
\hline Both & 4 & 26.6 & 6 & 28.6 & 6 & 50.0 & 0.6 & 0.4 \\
\hline Epilepsy syndrome: & & & & & & & & \\
\hline Idiopathic generalized epilepsy & 13 & 86.7 & 18 & 85.7 & 8 & 100 & 1.3 & 0.5 \\
\hline Idiopathic focal epilepsy & 2 & 13.3 & 3 & 14.3 & 0 & 0.0 & & \\
\hline Interictal EEG: & & & & & & & & \\
\hline Normal & 3 & 20.0 & 8 & 38.1 & 2 & 25.0 & 0.1 & -0.8 \\
\hline Focal & 5 & 33.3 & 4 & 19.0 & 3 & 37.5 & 0.1 & 0.8 \\
\hline Primary generalized & 6 & 40.0 & 5 & 23.8 & 2 & 25.0 & 1.3 & 0.2 \\
\hline Focal with secondary generalization & 1 & 6.7 & 4 & 19.0 & 1 & 12.5 & 0.1 & 0.8 \\
\hline
\end{tabular}

${ }^{*} \mathrm{p}<0.05$ is statistically significant.

Table 5. Binary logistic regression analysis of drug resistance within epileptic patients with significantly associated risk factors:

\begin{tabular}{|l|c|c|}
\hline & Odds ratio (95\% CI) & \multicolumn{1}{|c|}{ P value } \\
\hline No of seizures before treatment & $2.9(1.3-6.6)$ & $\mathbf{0 . 0 1 *}$ \\
\hline Interictal EEG Focal epileptogenic activity with secondary generalization & $5.8(2.3-14.6)$ & $0.97(0.82-1.1)$ \\
\hline Age of onset of epilepsy & $0.01 *$ \\
\hline Idiopathic focal epilepsy & $3.7(0.0-4.0)$ & 0.9 \\
\hline
\end{tabular}

${ }^{*} \mathrm{p}<0.05$ is statistically significant.

The distribution of different genotypes of C3435T polymorphism was compared between our study groups and crude odds ratio revealed a lower risk of drug resistance in patients with the genotype CT (OR: 0.8) than the genotypes CC and TT (OR: 1.2, 1 respectively) but the results were not statistically significant (Table 2, Table 3). Genotype CC was statistically more prevalent with seizures occurring during daytime than genotype CT and TT. However, genotype frequencies did not differ among those with focal or generalized epilepsy syndromes, family history, consanguinity, history of febrile seizures, history of status epilepticus and interictal EEG abnormalities (Table 4). Binary logistic regression analysis in Table 5 illustrated that higher number of seizures before treatment and interictal epileptogenic activity in the form of focal discharge followed by secondary generalization were significantly related (independent variables) to drug resistance among idiopathic epileptic patients. 


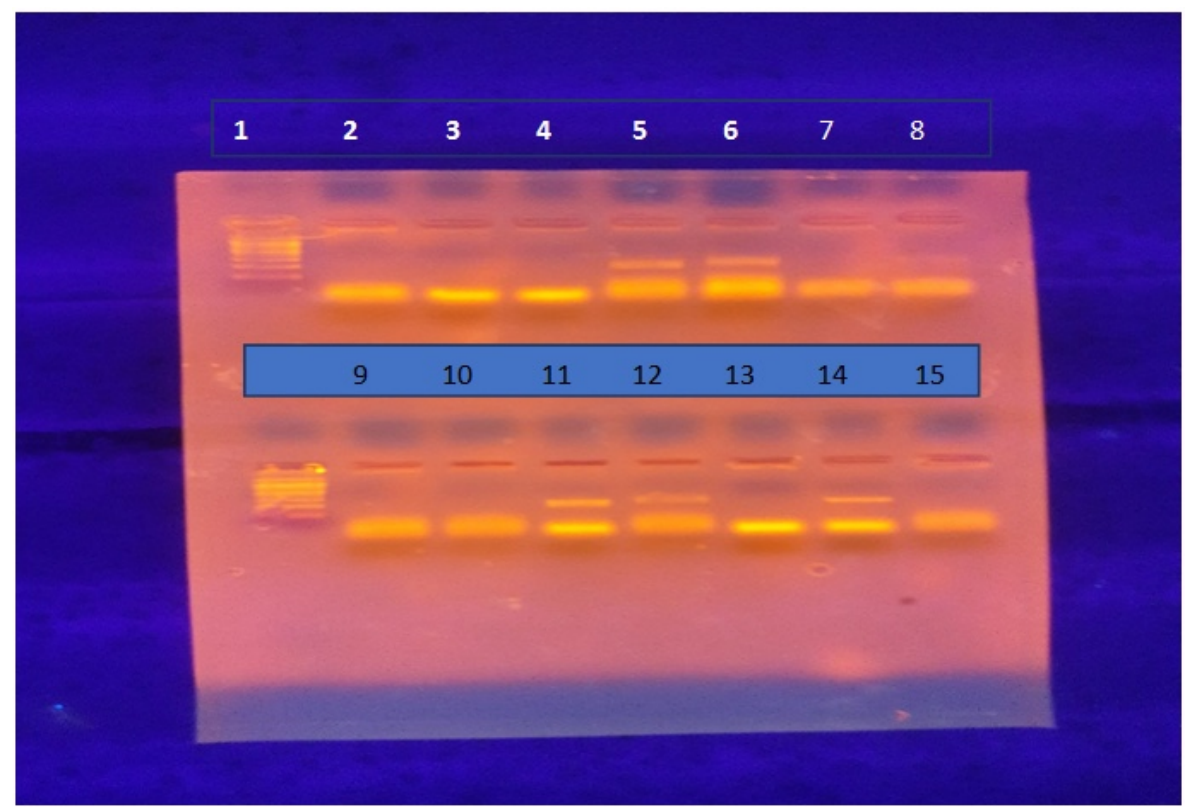

Figure 1. PCR amplification of 3435 C/T SNP of Egyptian epileptic patients and healthy control with agarose gel electrophoresis. lane 1:1000 bp DNA ladder; lane 5,6,11,12,14 heterozygous CT genotype; lane 3,4,13 homozygous CC genotype and lanes 2,7,8,9,10,15 homozygous TT genotype

\section{Discussion}

The mechanisms underlying resistance to therapy by AEDs have not well been established. Underlying structural abnormalities within the brain might play a role [4]. However, up till now response to therapy differs from one individual to another even within the same syndrome. This encouraged researchers to search for another explanation to the phenomenon underlying drug resistance. The pharmacokinetic hypothesis gained much attention during past years. Researchers showed that entry of AEDs to the epileptogenic zone across BBB might be impaired by drug efflux protein transporter P-gp, whose overexpression was suggested to be modified by genetic polymorphism of its coding region on exon 26 C3435T [6,7].

In the present study, we performed a genetic evaluation of polymorphism of the C3435T gene in order to define its association with drug resistance among idiopathic epileptic Egyptian patients.

Our work found a lower risk of drug resistance in patients with genotype CT than the genotype CC and TT. However, the distribution of genotypes was not significant.

Initial report of association between C3435T polymorphism and pharmacoresistance in epileptic patients was reported by Siddiqui et al [5]. He found that homozygosity 3435C may be related to drug resistance. Some studies go in hand with his observations [11,12,13]. However subsequent studies had provided conflicting results and failed to prove the original findings [14,15,16,17]. Moreover, other studies on different ethnic populations reported the opposite with 3435T homozygosity was the accused of resistance $[18,19,20]$. Sayyah and colleagues found in their study on idiopathic Iranian epileptic patients that there were more patients with CT and TT genotypes among those with lower number of seizures, and more patients with CC genotype among those with higher number of seizures [21].

In our work, the analysis of the genotypes with some data of epilepsy including family history, consanguinity, history of febrile seizures, history of status epilepticus showed no statistically significant difference. Also, the association of genotypes with epilepsy syndrome and interictal EEG finding showed similar results. Daytime seizures were significant among CC genotype in comparison to CT and TT genotypes. Up to our knowledge, this result is not documented before.

\section{Conclusion}

Whether SNP C3435T of ABCB1 gene is associated with the risk of pharmacoresistance in epilepsy or not remains to be elucidated. Our work hypothesized possible role of ABCB1 C3435T polymorphism in pharmacoresistant epilepsy. We found that low risk to pharmacoresistance was associated with those with CT genotype, though this association was not significant due to some limitations including small sample size of our study, using different AEDs which may not be P-gp substrates. So, larger studies with large sample of populations, focusing on single AED proved to be substrate of P-gp should be done. Also, different polymorphisms are still needed to be studied to illustrate the association between genotypes and pharmacoresistance.

\section{List of Abbreviations}

AEDs, Antiepileptic drugs; ABCB1, ATP binding cassette subfamily B member 1; BBB, Blood brain barrier; EEE, Electroencephalography; P-gp, P-glycoprotein; PCR-RFLP, polymerase chain reaction-restriction fragment length polymorphism

\section{Conflict of Interest}

The authors declare that they have no competing interests. 


\section{Financial Support}

There is no source of funding for the research.

\section{References}

[1] Jessica, J.F., Ingrid, E.S., Robert, S.F, "The new definition and classification of seizures and epilepsy," Epilepsy Research, 139. 73-79. 2018.

[2] Panayiotopoulos, C.P, Syndromes of idiopathic generalized epilepsies not recognized by the International League against Epilepsy. Epilepsia, 2005, 46. 57-66.

[3] Schmidt, D. and Loscher, W, "Drug resistance in epilepsy: putative neurobiologic and clinical mechanisms," Epilepsia, 46 (6). 858-877. 2005.

[4] Boonluksiri, P., Visuthibhan, A. and Katanyuwong, K, "Clinical Prediction Rule of Drug Resistant Epilepsy in Children," Journal of epilepsy research, 5(2). 84-88. 2015.

[5] Siddiqui, A., Kerb, R., Weale, ME., Brinkmann, U., Smith, A., Goldstein, D.B., Wood, N.W. and Sisodiya, S.M," Association of multidrug resistance in epilepsy with a polymorphism in the drugtransporter gene ABCB1," New England Journal of Medicine, 348(15). 1442-1448. 2003.

[6] Manna, J. and Sanjeev, T, "Role of multidrug transporters in neurotheraputics," Annals of Indian Academy of Neurology, 12(2). 89-98. 2009.

[7] Komar, A.A, "Silent SNPs: impact on gene function and phenotype," Pharmacogenomics, 8(8). 1075-1080. 2007.

[8] Kwan, P., Arzimanoglou, A., Berg, A., Brodie, M., Hauser, W. Mathern, G., Perucca, S., Wiebe, S. and French, J, "Definition of drug resistant epilepsy: Consensus proposal by the ad hoc Task Force of the ILAE Commission on Therapeutic Strategies," Epilepsia, 51(6).1069-1077. 2010.

[9] Morisky, D.E., Green, L.W. and Levine, D.M, "Concurrent and predictive validity of a self-reported measure of medication adherence," Medical Care, 24. 67-74. 1986.

[10] Emich-Widera, E., Likus, W., Kazek, B., Sieroń, A. and Urbanek, $\mathrm{K}$, "Polymorphism of ABCB1/MDR1 C3435T in Children and Adolescents with Partial Epilepsy is due to Different Criteria for Drug Resistance - Preliminary Results, " Medical Science Monitor, 20. 1654-1661. 2014.

[11] Zimprich, F., Sunder-Plassmann R., Stogmann, E., Gleiss, A., Dal-Bianco , A., Zimprich, A., Plumer, S., Baumgartner, C. and Mannhalter, C, "Association of an ABCB1 gene haplotype with pharmacoresistance in temporal lobe epilepsy," Neurology, 63.1087-1089. 2004.

[12] Hung, C.C., Tai, J.J., Lin, C.J., Lee, M.J. and Liou, H.H, "Complex haplotypic effects of the ABCB1 gene on epilepsy treatment response," Pharmacogenomics, 6. 411-417. 2005.

[13] Ebid, A.H., Ahmed, M.M. and Mohammed, S.A,"Therapeutic drug monitoring and clinical outcomes in epileptic Egyptian patients: a gene polymorphism perspective study," Therapeutic Drug Monitoring, 29(3). 305-312. 2007.

[14] Dericioglu, N., Babaoglu, M.O., Yasar, U., Bal, I.B., Bozkurt, A. and Saygi, S, "Multidrug resistance in patients undergoing resective epilepsy surgery is not associated with C3435T polymorphism in the ABCB1 (MDR1) gene," Epilepsy Research, 80. 42-46. 2008

[15] Ozgon, G.O., Bebek, N., Gul, G. and Cine, N, "Association of MDR1 (C3435T) polymorphism and resistance to carbamazepine in epileptic patients from Turkey," European Neurology, 59(1-2). 67-70. 2008.

[16] Shahwana, A., Murphya, K., Dohertyc, C., Cavalleri, G., Muckiane, C., Dickere, P., McCarthya, M., Kinironsa, P., Goldsteind, D. and Delantya, N,"The controversial association of ABCB1 polymorphisms in refractory epilepsy: An analysis of multiple SNPs in an Irish population, " Epilepsy Research, 73. 192-198. 2007.

[17] Kim, D.W., Kim, M., Lee, S.K., Kang, R. and Lee, S.Y, "Lack of association between C3435T nucleotide MDR1 genetic polymorphism and multidrug-resistant epilepsy," Seizure, 15. 344-347. 2006.

[18] Seo, T., Ishitsu, T., Ueda, N., Nakada, N., Yurube, K., Ueda, K. and Nakagawa, K, "ABCB1 polymorphisms influence the response to antiepileptic drugs in Japanese epilepsy patients," Pharmacogenomics, 7(4). 551-561. 2006.

[19] Ponnala, S., Chaudhari, J.R., Jaleel, M.A., Bhiladvala, D., Kaipa, P.R., Das, U.N. and Hasan, Q, "Role of MDR1 C3435T and GABRG2 C588T gene polymorphisms in seizure occurrence and MDR1 effect on antiepileptic drug (phenytoin) absorption," Genetic Testing and Molecular Biomarkers, 16. 550-557. 2012.

[20] Shaheen, U., Prasad, D.K., Sharma, V., Suryaprabha, T., Ahuja, Y.R., Jyothy, A. and Munshi, A, "Significance of MDR1 gene polymorphism C3435T in predicting drug response in epilepsy," Epilepsy Research, 108(2). 251-256. 2013

[21] Sayyah, M., Kamgarpour, F., Maleki, M., Karimipoor, M., Gharagozli, K. and Shamshiri, A.R, "Association analysis of intractable epilepsy with C3435T and G2677T/A ABCB1 gene polymorphisms in Iranian patients," Epileptic Disorder, 13(2). 155-165. 2011.

(C) The Author(s) 2019. This article is an open access article distributed under the terms and conditions of the Creative Commons Attribution (CC BY) license (http://creativecommons.org/licenses/by/4.0/). 